\title{
COMPLETE PATHOLOGICAL RESPONSE AFTER NEOADJUVANT CHEMOTHERAPY IN BREAST CANCER PATIENTS: ANALYSIS OF 83 PATIENTS TREATED AT A FEDERAL PUBLIC SERVICE
}

Maria Gabriela Ferreira Silva, Isabella Cristina Santos Soares, Flávia Pinto Cardozo', Flávia Maria Souza Clímaco, Aguinaldo Ferreira Leite Filho'

${ }^{1}$ Hospital Federal de Ipanema - Rio de Janeiro (RJ), Brazil.

Introduction: Neoadjuvant chemotherapy is performed before surgical treatment and aims to make a locally advanced tumor operable, provide conservative treatment, demonstrate tumor sensitivity in vivo and evaluate the pathological response (PR) to treatment. PR is an important prognostic factor, and several studies have demonstrated a correlation between the biological factors of the tumor and its response rate. Patients with a pathologic complete response (PCR) present a longer survival rate compared to those with residual disease. Objectives: The main objective was to assess PCR in patients with breast cancer undergoing neoadjuvant chemotherapy. As secondary objectives, we identified clinical-pathological variables related to PCR and correlated the pathologic response in the breast with the axillary response after chemotherapy. Methods: Four hundred and forty-four medical records of patients seen in the Mastology sector were reviewed between January 2016 and July 2019. Eighty-three patients were selected, with 361 cases that did not meet the inclusion criteria being excluded. The exclusion criteria were benign disease, neoadjuvant hormone therapy, neoadjuvant radiation therapy, trastuzumab associated with chemotherapy, upfront surgery and patients who did not receive surgical treatment after chemotherapy. The variables analyzed were age, tumor size, axillary involvement, histological type and grade, molecular subtype and PCR. Results: Most patients were over 50 years of age (62\%) and had tumors larger than $5 \mathrm{~cm}(75 \%)$. Fifty of them (60\%) had initial axillary involvement. Among the 83 patients, 64 (77\%) did not obtain a pathologic response in the breast and armpit. Two (3\%) showed a response only in the breast. PCR was observed in 17 patients (20\%) and almost half of them were under 50 years of age (47\%). Moderately differentiated (grade 2) and undifferentiated (grade 3) tumors, accounted for $96 \%$ of cases and had a higher rate ofPCR than grade 1 tumors. In HER2 positive subtypes, PCR occurred in $36 \%$ and in negative triples in $22 \%$, being higher than in luminous A and B subtypes (15\% and 17\%, respectively). Conclusions: The histological grade and molecular subtypes correlate with the pathologic response to neoadjuvant chemotherapy. More undifferentiated tumors and the triple negative and HER 2 positive molecular subtypes have a higher PCR rate. Despite the small sample, the results of this study were similar to those in the medical literature. A higher number of cases is necessary to corroborate the data obtained, as well as a longer follow-up time to demonstrate the relationship between PCR and survival. 